\title{
Cell cycle progression score predicts metastatic progression of clear cell renal cell carcinoma after resection
}

\author{
Eric J. Askeland ${ }^{\mathrm{a}}$, Vincent A. Chehval ${ }^{\mathrm{a}}$, Ryan W. Askeland ${ }^{\mathrm{b}}$, Placede G. Fosso ${ }^{\mathrm{c}}$, Zaina Sangale ${ }^{\mathrm{c}}$, \\ Nafei Xuc ${ }^{\mathrm{c}}$, Saradha Rajamanic ${ }^{\mathrm{c}}$, Steven Stone ${ }^{\mathrm{c}}$ and James A. Brown ${ }^{\mathrm{a}, *}$ \\ ${ }^{a}$ Department of Urology, University of Iowa, IA, USA \\ ${ }^{\mathrm{b}}$ Department of Pathology, University of Iowa, IA, USA \\ ${ }^{\mathrm{c}}$ Myriad Genetics, University of Iowa, IA, USA
}

\begin{abstract}
.
BACKGROUND: The outcome of surgically resected, apparently localized, clear cell renal carcinoma (ccRCC) is uncertain. OBJECTIVE: To evaluate if cell cycle progression (CCP) gene expression can predict future metastasis.

METHODS: Pathologic T2a-T3b tumors at University of Iowa were reviewed. Patients with known or suspected metastasis, lymph node involvement or who received neoadjuvant or adjuvant radiation, chemotherapy or immunotherapy were excluded. Case and control cohorts were defined as those who did or did not develop metastatic disease within 5 years. Measured levels of 31 cell cycle genes and 15 control genes from the tumor were calculated as a CCP score. Additionally, gene expression data for a separate ccRCC cohort was downloaded from The Cancer Genome Atlas (TCGA).

RESULTS: Univariate analysis of 26 cases and 38 controls revealed that the CCP score predicted progression to metastasis (OR 2.65, $p=0.0091)$. In multivariate logistic regression modeling, CCP expression remained a significant independent predictor for progression $(p=0.026)$. The CCP score was also significantly associated with distant metastasis in the TCGA renal cancer cohort in both univariate $\left(p=1.0 \times 10^{-9}\right)$ and multivariate $\left(p=5.6 \times 10^{-3}\right)$ analysis.

CONCLUSION: The CCP score has prognostic value in predicting metastatic progression after resection of organ-confined
\end{abstract} ccRCC.

Keywords: Clear cell renal cell carcinoma, kidney cancer, metastasis, biological markers

\section{Introduction}

Kidney cancer accounts for approximately $2 \%$ of all malignancies, and approximately $60 \%$ are localized disease at the time of diagnosis [12]. Unfortunately, approximately $30 \%$ of clear cell renal cell carcinomas (ccRCC) develop metastatic disease following surgical intervention with curative intent. Metastatic

* Corresponding author: James A. Brown, Department of Urology, University of Iowa, 200 Hawkins Dr., 3 RCP, Iowa City, IA 522421089, USA. Tel.: +1 319384 7295; Fax: +1 319356 3900; E-mail: james-brown-2@uiowa.edu.
ccRCC is refractory to traditional chemoradiation and historically the only effective systemic therapy was immunotherapy [3]. Newer therapies targeting the VEGF pathway, such as bevacizumab, tyrosine kinase inhibitors (TKIs) and mammalian target of rapamycin (mTOR) inhibitors, have emerged showing some benefit.

Although clinical variables (tumor size, grade, stage) provide useful information regarding the likelihood of disease progression, effort to identify a biomarker which identifies metastatic progression continues. Expression levels of cell cycle progression (CCP) genes have been found to be predictive of relapse or disease 
aggressiveness, first in breast and subsequently in several other types of cancers [4]. This includes prostate cancer patients after radical prostatectomy [5]6], after prostate biopsy [7] and conservative management, and after external beam radiation therapy [8]. Though prostate cancer and ccRCC are very different malignancies, cell cycle progression aberrations are common in neoplastic processes; thus, CCP gene expression profiles may also have prognostic value in ccRCC. Here we evaluated the prognostic utility of the CCP score in predicting progression to metastatic ccRCC after local resection of seemingly organ-confined disease. We performed an initial assessment of CCP expression in locally advanced renal cell carcinoma at risk for metastasis in the hopes the results would be hypothesis generating.

\section{Methods}

\subsection{Ethics statement}

This study was approved by the University of Iowa Institutional Review Board. The Institutional Review Board did not require written informed consent because this was a retrospective chart review. Patient identifying information was not included in analyses.

\subsection{Patients}

For the Iowa cohort study, we identified patients undergoing surgery between 1995, to maintain as modern a patient cohort as possible, and 2010, to allow for 5 years' follow-up. Two primary sources were used to identify patients eligible for study. An existing database was utilized that included all patients undergoing a partial nephrectomy or radical nephrectomy for neoplastic or benign conditions at the University of Iowa between 2000 and 2010. In total 1,131 patient charts were reviewed using this database. To further identify additional eligible patients between 19952000 all pathology records at our institution were searched using the search term "renal cell carcinoma". In total, 197 pathology records were reviewed for patients in this time period and a chart review was initiated on patients with pathologic findings meeting the criteria listed below. All patients with benign pathology or malignancy other than clear cell renal cell carcinoma were excluded. Only patients with pathologic T-stage T2a-T3b disease were included. Those with known or suspected metastasis prior to tumor resec- tion were excluded, as were those with lymph node involvement found at the time of resection. Further, patients who received neoadjuvant or adjuvant radiation, chemotherapy or immunotherapy were also excluded. A positive surgical margin was an exclusion criteria for the case cohort. Follow-up year was defined by rounding follow-up date to nearest year from anniversary of surgery. The significant majority of the T2a-T3b patients did have evidence of metastatic disease at time of surgery, a positive surgical margin or received neoadjuvant or adjuvant therapy, leaving 68 patients ( 28 cases, 40 controls) who met all inclusion criteria.

\subsection{Sample preparation}

Formalin-fixed paraffin-embedded (FFPE) blocks containing kidney tissue following nephrectomy for selected patients were pulled from the pathology repository. Histologic analysis, including FNG, was independently re-reviewed by a single pathologist. A total of six unstained slides were prepared and sent to Myriad Genetics, Inc. for analysis. The first slide was stained with hematoxylin and eosin for evaluation of tumor content and marking of tumor area as a guide for manual macrodissection of the remaining five slides. After macrodissection the tissue was placed in a microcentrifuge tube. Paraffin was removed using deparaffinization solution (Qiagen). Samples were treated for $2 \mathrm{~h}$ with proteinase $K$ digestion at $58^{\circ} \mathrm{C}$. Total RNA was extracted using miRNeasy FFPE kit (Qiagen) as described by the manufacturer.

\subsection{Real-time PCR}

Isolated total RNA was treated with DNase I (Sigma) prior to cDNA synthesis. The high-capacity cDNA Archive Kit (Applied Biosystems) was used to convert total RNA into single stranded cDNA as described by the manufacturer. Thirty-one cell cycle genes and 15 housekeeper control genes (HK genes) were selected based on Myriad's previous work. The sample cDNA was pre-amplified with a pooled reaction containing cell cycle and HK gene TaqMan assays. Preamplification reaction conditions were: $95^{\circ} \mathrm{C}(10 \mathrm{~min})$, followed by 14 cycles of $95^{\circ} \mathrm{C}$ ( 15 Section $60^{\circ} \mathrm{C}$ ( 4 min). Reactions were diluted 1:20 using the $1 \mathrm{XTE}$ buffer prior to loading on Taqman Low Density Arrays (TLDA, Applied Biosystems) to measure gene expression.

\subsection{RNA sequencing}

rRNA was depleted from total RNA by magnetic beads (Ribo-Zero kit) that selectively bind to rRNA- 
biotinylated probe duplexes. Resulting total RNA was converted to double stranded cDNA, then adaptor ligated and PCR amplified using TruSeq stranded total RNA sequencing kit per manufacturer recommendation. Normalized libraries were sequenced in Illumina HiSeq sequencer to obtain a read size of $50 \mathrm{bp}$. The average number of total reads per sample was 61,393 , 064. The average number of reads aligning to the transcript corresponding to the $75^{\text {th }}$ percentile in expression level was 20 .

\subsection{Cell cycle progression (CCP) score calculation}

When adequate material was present, CCP scores were derived from qRT-PCR and from next generation RNA sequencing data. The qRT-PCR CCP score was calculated, as previously described, from the expression of the data of the 31 CCP genes, which were normalized to 15 housekeeper genes [5]. Each was analyzed in triplicate and averaged. The sequencing CCP score was calculated as the mean of the normalized counts for the $31 \mathrm{CCP}$ genes. Normalization is performed by taking the base 2 logarithm of the counts divided by the $75^{\text {th }}$ percentile for each sample and run. Results with 0 counts are treated as missing.

\subsection{Statistical analysis}

\subsubsection{Iowa cohort}

Sex, age at surgery, TNM tumor stage, follow-up since surgery/time to metastasis, tumor size, FNG, lymphovascular invasion (LVI), smoking status and CCP scores were available for each patient and included in the analysis. This analysis evaluated the association of CCP scores, clinical parameters, and RNA expression levels of the entire transcriptome with metastatic progression of the cancer modeled as the response variable.

\subsubsection{TCGA cohort}

Cohort data was downloaded from the TCGA data portal (https://tcga-data.nci.nih.gov/tcga/tcgaDownlo ad.jsp). Gender, age at initial pathological diagnosis, collection site, race, T-stage, lymph node status, distant metastatic status and gene expression sequencing data were available for each patient. We tested the association of sequencing CCP score, RNA expression levels of the entire tumor transcriptome and clinical covariates with distant metastatic status.

We evaluated the prognostic value of $\mathrm{CCP}$, clinical variables, and transcriptome expression levels in terms

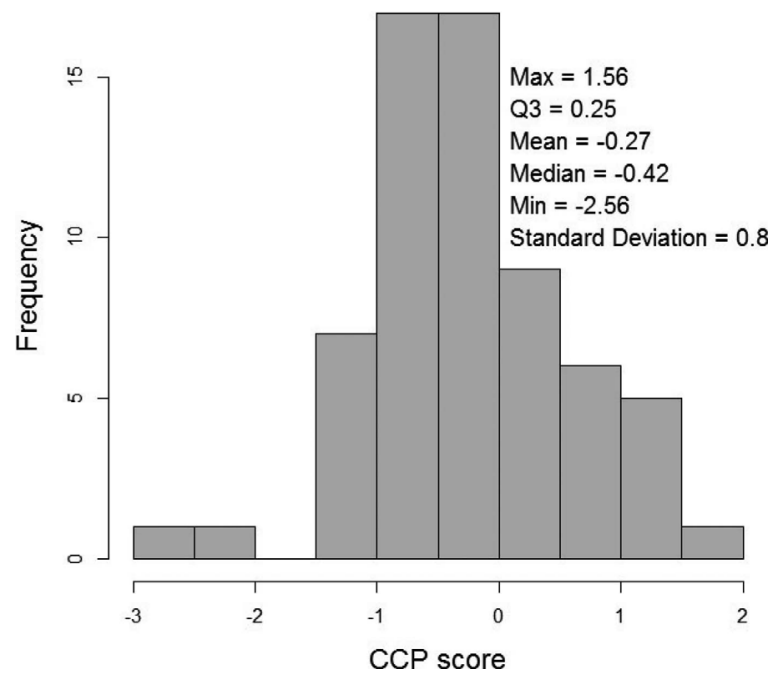

Fig. 1. Distribution of all CCP scores.

of $p$-values, adjusted for multiple testing when necessary, and odd-ratios from univariate and multivariate logistic regression models. The test statistic was the change in the likelihood deviance metric between the full model and the appropriate reduced model. Odds ratios were calculated to measure the risk of metastatic cancer for a one-unit increase in the corresponding variable. Statistical analyses were conducted using the $\mathrm{R}$ software environment (version 2.14.1, December 2011, $R$ Development Core Team) and SAS 9.2 (SAS Institute, Cary NC). $P$-values were considered significant at a two-sided significance level of 0.05 .

\section{Results}

We initially studied the Iowa cohort. A total of 40 and 28 patients met all inclusion criteria for the control and case groups, respectively, on initial screening. Four additional patients were subsequently excluded from further analysis. One case patient was excluded because further analysis revealed a positive margin, and three patients were excluded due to pathology demonstrating ccRCC with sarcomatoid differentiation. Sarcomatoid differentiation was not a pre-specified exclusion criteria, however, as sarcomatoid histology is strongly associated with aggressive and metastatic disease, these patient samples were removed from cohort comparison to avoid having undue influence on our analysis of CCP score as a predictor of disease progression.

The remaining 64 patients, 26 cases and 38 controls, were analyzed. CCP scores were calculated on 
Table 1

Patient characteristics

\begin{tabular}{|c|c|c|c|}
\hline & & Cases & Controls \\
\hline \multirow[t]{2}{*}{ Sex } & Female & $7(27 \%)$ & $16(42 \%)$ \\
\hline & Male & $19(73 \%)$ & $22(58 \%)$ \\
\hline \multirow{4}{*}{$\begin{array}{l}\text { Age at } \\
\text { surgery } \\
\text { (years) }\end{array}$} & Q1 & 55.85 & 50.22 \\
\hline & Median & 61.03 & 59.31 \\
\hline & Mean & 61.35 & 57.92 \\
\hline & Q3 & 69.58 & 64.96 \\
\hline \multirow[t]{2}{*}{ T stage } & II & $6(23 \%)$ & $17(45 \%)$ \\
\hline & III & $20(77 \%)$ & $21(55 \%)$ \\
\hline \multirow{4}{*}{$\begin{array}{l}\text { Time to } \\
\text { metastasis } \\
\text { (years) }\end{array}$} & Q1 & 1.06 & \\
\hline & Median & 1.68 & \\
\hline & Mean & 2.65 & \\
\hline & Q3 & 3.69 & \\
\hline \multirow{4}{*}{$\begin{array}{l}\text { Last follow up } \\
\text { since } \\
\text { surgery } \\
\text { (years) }\end{array}$} & Q1 & & 5.88 \\
\hline & Median & & 6.69 \\
\hline & Mean & & 7.76 \\
\hline & Q3 & & 9.28 \\
\hline \multirow[t]{2}{*}{ Surgery } & Partial nephrectomy & $0(0 \%)$ & $2(5 \%)$ \\
\hline & Radical nephrectomy & $26(100 \%)$ & $36(95 \%)$ \\
\hline Surgical & Positive & $0(0 \%)$ & $1(3 \%)$ \\
\hline Margins & Not involved & $26(100 \%)$ & $37(97 \%)$ \\
\hline \multirow{4}{*}{$\begin{array}{l}\text { Tumor } \\
\text { size }(\mathrm{cm})\end{array}$} & Q1 & 7.5 & 6.4 \\
\hline & Median & 9.75 & 8 \\
\hline & Mean & 9.48 & 7.84 \\
\hline & Q3 & 11 & 9.5 \\
\hline \multirow{2}{*}{$\begin{array}{l}\text { Fuhrman nuclear } \\
\text { grade (FNG) }\end{array}$} & Low grade (FNG I/II) & $7(27 \%)$ & $23(61 \%)$ \\
\hline & High grade (FNG III/IV) & $19(73 \%)$ & $15(39 \%)$ \\
\hline \multirow{2}{*}{$\begin{array}{l}\text { Lymphovascular } \\
\text { invasion }\end{array}$} & Present & $17(65 \%)$ & $11(29 \%)$ \\
\hline & Not present & $9(35 \%)$ & $27(71 \%)$ \\
\hline \multirow{2}{*}{$\begin{array}{l}\text { History of } \\
\text { smoking }\end{array}$} & Yes & $14(54 \%)$ & $21(55 \%)$ \\
\hline & No & $12(46 \%)$ & $17(45 \%)$ \\
\hline \multirow[t]{4}{*}{ CCP score } & Q1 & -0.49 & -0.84 \\
\hline & Median & -0.11 & -0.52 \\
\hline & Mean & 0.075 & -0.50 \\
\hline & Q3 & 0.76 & -0.13 \\
\hline
\end{tabular}

all specimens. Figure 1 shows the distribution of CCP scores, which approximated a bell-shaped curve. Demographic data for this cohort is presented in Table 1. Median age was 61.0 years (IQR 13.73) for the case cohort and 59.3 years (IQR 14.74) for the control cohort. The case group tended to have a higher TNM stage than the controls. Likewise, the case arm tended to have larger tumors and higher grade histology. The median time to metastasis in the case group was 1.68 years (IQR 2.63). The median follow-up for the control group was 6.69 years (IQR 3.4). Three control patients developed metastasis at $8.44,8.61$ and 9.28 years but were retained in the control group as the initial study design was to identify if CCP score is a predictor of metastasis within 5 years. One control patient was found to have a positive margin at the time of surgical resection. However, the patient has never developed disease progression and was included in the
Table 2

Univariate logistic regression model for clinical variables and $\mathrm{CCP}$ score demonstrating differences between the control cohort and the metastatic cohort

\begin{tabular}{lcclc}
\hline Variable & $\begin{array}{c}\text { Odds } \\
\text { ratio (OR) }\end{array}$ & $\begin{array}{c}95 \% \text { CI } \\
\text { for OR }\end{array}$ & P-value & AUC \\
\hline $\begin{array}{l}\text { Lymphovascular } \\
\text { invasion }\end{array}$ & 4.64 & $(1.63,14.082)$ & 0.0050 & 0.68 \\
$\quad$ Yes vs. No) & & & & \\
CCP score & 2.65 & $(1.34,5.89)$ & 0.0091 & 0.68 \\
Fuhrman nuclear & 4.16 & $(1.46,12.97)$ & 0.0099 & 0.67 \\
$\quad$ grade & & & & \\
$\quad$ (High vs. Low) & & & & \\
Tumor size & 1.19 & $(1.0075,1.44)$ & 0.052 & 0.66 \\
$\quad$ TNM T-stage & 2.69 & $(0.91,8.75)$ & 0.081 & 0.61 \\
$\quad$ III vs. II) & & & & \\
Age & 1.037 & $(0.98,1.097)$ & 0.18 & 0.60 \\
Sex (Male & 1.97 & $(0.68,6.08)$ & 0.22 & 0.58 \\
$\quad$ vs. Female) & & & & \\
Smoking & 0.94 & $(0.34,2.59)$ & 0.91 & 0.51 \\
$\quad$ Yes vs. No) & & & & \\
\hline
\end{tabular}

Table 3

Multivariate logistic regression model with age, tumor size, CCP score, lymphovascular invasion, TNM T-stage, Fuhrman nuclear grade, and smoking as covariates demonstrating differences between the control cohort and the metastatic cohort

\begin{tabular}{lccl}
\hline Variable & $\begin{array}{c}\text { Odds } \\
\text { ratio (OR) }\end{array}$ & $\begin{array}{c}95 \% \text { CI } \\
\text { for OR }\end{array}$ & P-value \\
\hline Age & 1.12 & $(1.04,1.23)$ & 0.0045 \\
Tumor size & 1.34 & $(1.06,1.78)$ & 0.022 \\
CCP score & 3.40 & $(1.24,11.27)$ & 0.026 \\
$\quad$ Lymphovascular & 3.13 & $(0.68,15.55)$ & 0.14 \\
$\quad$ invasion (Yes vs. No) & & & \\
Sex (Male vs. Female) & 3.15 & $(0.61,20.82)$ & 0.19 \\
Stage (III vs. II) & 2.48 & $(0.49,13.61)$ & 0.27 \\
Fuhrman nuclear & 1.76 & $(0.36,8.78)$ & 0.47 \\
$\quad$ grade (High vs. Low) & & & \\
$\quad$ Smoking (Yes vs. No) & 0.65 & $(0.14,2.85)$ & 0.57 \\
\hline
\end{tabular}

Table 4

Multivariate logistic regression modeling using step-wise variable selection. The covariates retained in the model are age, CCP score, tumor size and lymphovascular invasion

\begin{tabular}{lccl}
\hline Variable & $\begin{array}{c}\text { Odds } \\
\text { ratio (OR) }\end{array}$ & $\begin{array}{c}\text { 95\% CI } \\
\text { for OR }\end{array}$ & $P$-value \\
\hline Age & 1.11 & $(1.03,1.19)$ & 0.0057 \\
CCP score & 3.89 & $(1.44,10.47)$ & 0.0072 \\
Tumor size & 1.26 & $(1.003,1.56)$ & 0.047 \\
Lymphovascular & 4.46 & $(1.24,16.03)$ & 0.022 \\
$\quad$ invasion (Yes vs. No) & & & \\
\hline
\end{tabular}

control arm for statistical comparison. The mean CCP score for the case group was 0.075 compared to -0.50 for the control arm.

In univariate logistic regression modeling, only LVI, CCP score and FNG were statistically significant predictors for development of metastasis (Table 2). Odds 
Table 5

41 genes associated with metastatic disease in TCGA cohort. CCP genes are in bolded

\begin{tabular}{|c|c|c|c|c|c|c|c|c|}
\hline IL20RB & BIRC5 & PCK1 & TROAP & GTSE1 & CDC20 & ACVR2A & TSHZ1 & PRCKE \\
\hline CTSG & TMEM150C & TGF2BP3 & SKA1 & MIIP & CCNB2 & UBE2C & KIF18B & RGS17 \\
\hline CENPA & PTTG1 & HJURP & APOL2 & TSPAN7 & NCAPG & CDCA3 & RAD54L & KIF2C \\
\hline ENPP5 & PBX1 & SHE & MOV10 & CEP55 & ARHGAP24 & LOC100128191 & MYBL2 & PT-PRB \\
\hline INHBE & C22orf9 & PARP12 & ITPKA & KIF 20A & & & & \\
\hline
\end{tabular}

ratio was greatest for LVI at 4.64 (95\% CI $1.63-$ 14.082). Tumor size, T-stage, age, gender and smoking were not statistically significant in this analysis.

In multivariate logistic regression modeling, only age, tumor size and CCP score were significantly associated with progression to metastatic disease (Table 3). Odds ratio was greatest for CCP score at $3.40(95 \%$ CI 1.24-11.27). LVI, gender, T-stage, FNG and smoking status were not statistically significant. We used stepwise variable selection to build the best model which ultimately included patient age, CCP score, tumor size and LVI (Table 4). An AUC of 0.84 was obtained with these variables, which decreased to 0.78 if CCP score was excluded.

The three patients with sarcomatoid differentiation were excluded from the above statistical comparisons. However, these specimens with sarcomatoid differentiation did have very high CCP scores, including the two highest scores observed in this study (2.45 and 2.41). The CCP score of the third patient was also relatively high at 1.03 , consistent with the hypothesis that CCP score might identify aggressive malignant transformation.

To determine if other genes were associated with eventual development of metastatic disease, we evaluated the RNA expression levels of the entire tumor transcriptome from the patients in Iowa case/control series using RNA sequencing protocols on 'next generation' sequencing platforms. The CCP scores calculated from sequencing data were highly correlated with the scores derived from qRT-PCR (Pearson correlation coefficient 0.91). With the exception of the CCP genes ( $p$-value for CCP score $=0.0016$ ), no other genes were significantly associated with disease outcome.

Next, we analyzed The Cancer Genome Atlas (TCGA) cohort. We attempted to identify prognostic RNAs in the publically available expression data from TCGA consortium. The clinical characteristics of this cohort have been described previously [9]. Using distant metastasis as the endpoint ( $N=466,72$ events) in a case/control study design, we found that $\mathrm{CCP}$ score was highly prognostic $(O R=2.2795 \% \mathrm{CI}(1.73$, $\left.2.35), p=1.0 \times 10^{-9}\right)$. This association remained significant after adjusting for clinical variables including

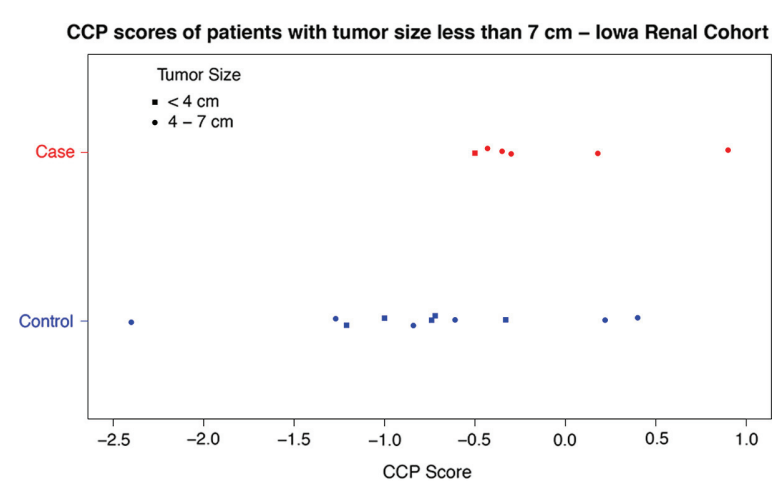

Fig. 2. CCP scores of patients with $<7 \mathrm{~cm}$ tumors. Cases are red and controls are blue. The points are staggered to avoid overlap. (Colours are visible in the online version of the article; http://dx.doi.org/10. 3233/CBM-150530)

pathologic stage, nodal status, and patient age $(O R=$ $\left.1.6495 \% \mathrm{CI}(1.17,3.03), p=5.6 \times 10^{-3}\right)$. This analysis was also adjusted for collection site since we found this variable to be associated with outcome ( $p$-value for site $=0.0093$ ). Finally, we looked for other prognostic genes in this data set. After adjusting for multiple testing (and collection site), we found significant associations for 41 genes (Table 5). Interestingly, 18 of these genes are CCP genes. None of the 41 genes remained significantly associated with metastatic disease after adjusting for the CCP score.

Lastly, we made an initial assessment of the potential utility of CCP score as prognostic biomarker test for small renal masses in the University of Iowa cohort, we identified and compared all 17 (16 pathologic stage T3a and 1 pathologic stage T3b) tumors less than $7 \mathrm{~cm}$, 5 cases and 12 control patients. All $5(100 \%)$ cases and 4 of $12(33 \%)$ control patients had CCP scores of -0.5 or greater (Fig. 2).

\section{Discussion}

The CCP score, derived from a prespecified and commercially available assay, was predictive of metastasis development within 5 years in a cohort consisting of pathologic stage T2a-T3b patients treated by nephrectomy for localized ccRCC at the University of 
Iowa. The CCP score also had prognostic utility in a second TCGA renal cancer cohort with metastatic disease (M1) at time of surgery [9]. In neither cohort were we able to identify genes significantly associated with outcome after adjusting for CCP score and other clinical variables. This suggests that CCP gene expression appears to be a dominant factor in determining disease aggressiveness, but because the cohorts in this study were relatively small, it remains possible that other genes besides CCP genes could contribute meaningful prognostic information. Because the assay used here was originally derived and tested in prostate cancer, the ideal gene set for ccRCC may be somewhat different from the genes evaluated in this study.

Other cell cycle based markers, including KI-67, p53, p21, MCM2, spermine and cyclin D1, have been studied in ccRCC [10-13]. Other researchers have developed combinations of markers and expression arrays [14-17]. While the prognostic performance of these gene panels was generally better than individual makers, they have, to date, not made significant clinical impact due to lack of validation or apparent added value beyond traditional clinicopathologic features.

Because histologic tumor features and clinical characteristics are unable to completely predict progression to metastatic disease in ccRCC patients, CCP gene testing may have clinical benefit. It might aid clinicians in identifying high-risk patients at risk for metastasis after surgical resection and further could guide choice of administration of medical therapies for high-risk patients.

The current CCP score may also have the potential to be a future useful biomarker for patients considering active surveillance, which is now considered even in select patients with clinical T1b and clinical T2 tumors [1819]. While tumor size, histology, grade and growth kinetics have been the key features used to best predict risk for metastasis of patients on active surveillance, there remains some concern regarding intraobserver and interobserver reproducibility.

This study has several limitations, including its retrospective nature and the small number of Iowa cohort patients. The former was due to our decision to study pathologic T2a-T3b tumor patients, the majority of which had evidence or suspicion for metastasis on imaging preoperatively, had node positive disease or positive surgical margins, had received neoadjuvant or adjuvant chemoradiotherapy or immunotherapy or were lost to follow-up within five years. The latter was in part due to our center's rural location and travel distance for many patients who elected to transfer their care.
In summary, this study provides evidence that the CCP score may be useful for evaluating the metastatic potential of large or locally extensive renal masses after surgical resection. However, given the recent paradigm shift toward surveillance of small renal masses, future assessment of the potential utility of CCP score in this clinical space is also warranted.

\section{Acknowledgement}

Some of the results published here are, in whole or part, based upon data generated by the TCGA Research Network: http://cancergenome.nih.gov/.

\section{References}

[1] Cho E, Adami H, Lindblad P. Epidemiology of renal cell cancer. Hematol Oncol Clin North Am. 2011; 25: 651-665.

[2] Howlader N, Krapcho M, Neyman N et al., SEER Cancer Statistics Review, 1975-2009 (Vintage 2009 Populations). National Cancer Institute. Bethesda, MD. Available at http:// seer.cancer.gov/csr/1975_2009_pops09/. Accessed January 5, 2014.

[3] Pantuck AJ, Zisman A, Belldegrun A. The changing natural history of renal cell carcinoma. J Urol. 2001; 166: 1611-1623.

[4] Mosley JD, Keri RA. Cell cycle correlated genes dictate the prognostic power of breast cancer gene lists. BMC Med Genomics. 2008; 1: 11.

[5] Cuzick J, Swanson GP, Fisher G, Brothman AR, Berney DM, Reid JE et al. Prognostic value of an RNA expression signature derived from cell cycle proliferation genes in patients with prostate cancer: a retrospective study. Lancet Oncol. 2011; 12: 245-255.

[6] Cooperberg MR, Simko JP, Cowan JE, Reid JE, Djalilvand A, Bhatnagar S, et al. Validation or a cell-cycle progression gene panel to improve risk stratification in a contemporary prostatectomy cohort. J Clin Oncol. 2013; 31: 1428-1434.

[7] Cuzick J, Berney DM, Fisher G, Mesher D, Møller H, Reid JE et al. Prognostic value of a cell cycle progression signature for prostate cancer death in a conservatively managed needle biopsy cohort. Br J Cancer. 2012; 106: 1095-1099.

[8] Freedland SJ, Gerber L, Reid J, Welbourn W, Tikishvili E, Park J, et al. Prognostic utility of cell cycle progression score in men with prostate cancer after primary external beam radiation therapy. Int J Radiat Oncol Biol Phys. 2013; 86: 848853.

[9] The Cancer Genome Atlas Research Network. Comprehensive molecular characterization of clear cell renal cell carcinoma. Nature. 2003; 499: 43-49.

[10] Tunuguntla HS, Jorda M. Diagnostic and prognostic molecular markers in renal cell carcinoma. J Urol. 2008; 179: 20962102.

[11] Nogueira M, Kim HL. Molecular markers for predicting prognosis of renal cell carcinoma. Urol Oncol. 2008; 26: 113-124.

[12] Ngo TC, Wood CG, Karam JA. Biomarkers of renal cell carcinoma. Urol Oncol. 2014; 32: 243-251.

[13] Lima MS, Pereira RA, Costa RS, Tucci S, Dantas M, Muglia $\mathrm{VF}$, et al. The prognostic value of cyclin D1 in renal cell carcinoma. Int Urol Nephrol. 2014; 46: 905-913. 
[14] Sultmann H, von Heydebreck A, Huber W, Kuner R, Buness $\mathrm{A}$, Vogt $\mathrm{M}$, et al. Gene expression in kidney cancer is associated with cytogenetic abnormalities, metastasis formation and patient survival. Clin Cancer Res. 2005; 11: 646-655.

[15] Kosari F, Parker AS, Kube DM, Lohse CM, Leibovich BC, Blute ML, et al. Clear cell renal cell carcinoma: gene expression analyses identify a potential signature for tumor aggressiveness. Clin Cancer Res. 2005; 11: 5128-5139.

[16] Parker AS, Leibovich BC, Lohse CM, Sheinin Y, Kuntz SM, Eckel-Passow JE, et al., Development and evaluation of BioScore: a biomarker panel to enhance prognostic algorithms for clear cell renal cell carcinoma. Cancer. 2009; 115:
2092-2103.

[17] Wu X, Weng L, Li X, Guo C, Pal SK, Jin JM, et al, Identification of a 4-microRNA signature for clear cell renal cell carcinoma metastasis and prognosis. PLoS One. 2012; 7: e35661.

[18] Chawla SN, Crispen PL, Hanlon AL, Greenberg RE, Chen DY, Uzzo RG. The natural history of observed enhancing renal masses: meta-analysis and review of the world literature. J Urol. 2006; 175: 425-431.

[19] Mehrazin R, Smaldone MC, Kutikov A, Li T, Tomaszewski JJ, Canter DJ, et al. Growth kinetics and short term outcomes of cT1b and cT2 renal masses under active surveillance. J Urol. 2014; 192: 659-664. 